\title{
I I 8 Real-time assessment of right and left ventricular volumes and function in patients with congenital heart disease using high spatio-temporal resolution radial $k$-t SENSE Vivek Muthurangu*, Philip Lurz, Andrew Taylor and Michael Hansen
}

Address: Institute of Child Health, London, UK

* Corresponding author

from I th $^{\text {th }}$ Annual SCMR Scientific Sessions

Los Angeles, CA, USA. I-3 February 2008

Published: 22 October 2008

Journal of Cardiovascular Magnetic Resonance 2008, I0(Suppl I):A243 doi:I0.I I86/I532-429X-I0-SI-A243

This abstract is available from: http://jcmr-online.com/content/I0/SI/A243

C 2008 Muthurangu et al; licensee BioMed Central Ltd.

\section{Introduction}

Magnetic resonance assessment of ventricular volumes and function has become an integral part of clinical management. However, traditional ECG gated, breath-hold, cine imaging is limited by long acquisition times, difficulty performing multiple breath-holds, and irregular heart rate. Another approach is real-time MR imaging, which can be performed without cardiac gating or breath holds. However, these benefits come at the cost of lower spatiotemporal resolution, which may affect accuracy. A solution is radial k-t SENSE which allows high spatiotemporal resolution real-time imaging to be performed.

\section{Purpose}

To compare ventricular volumes measured using (i) a cardiac gated sequence, (ii) a standard product real-time sequence, and (iii) a radial real-time k-t SENSE sequence in patients with congenital heart disease.

\section{Methods}

Forty consecutive children and adults with congenital heart disease were enrolled into this study (median age 23.7, full range 12.4-63.9 yrs, 21 males, 19 females). Ventricular volume assessment was performed using; (i) a Cartesian 2D, multi-slice, retrospectively cardiac gated, SSFP sequence (spatial resolution $1.8 \times 1.8 \times 10 \mathrm{~mm}$, temporal resolution $40 \mathrm{~ms}$ ), (ii) a standard Cartesian realtime 2D multi-slice SSFP sequence. (spatial resolution 2.7 $\times 3.3 \times 10 \mathrm{~mm}$, temporal resolution $80 \mathrm{~ms}$ ) and (iii) a radial real-time $2 \mathrm{D}$ multi-slice SSFP sequence accelerated with k-t SENSE. (spatial resolution $2.3 \times 2.3 \times 10 \mathrm{~mm}$, temporal resolution $40 \mathrm{~ms}$ ). Global image quality and motion fidelity was scored and compared with a Wilcoxon sign rank test. Image contrast, edge sharpness and summed perimeters were quantified, and compared using paired t-tests. Ventricular volumes were compared with paired t-tests, Bland Altman analysis and correlation coefficients.

\section{Results}

Global image quality, motion fidelity, image contrast, edge sharpness and summed perimeters were all greater for radial real-time k-t SENSE compared to standard realtime $(\mathrm{p}<0.05)$. However, the gated acquisitions were still superior to radial real-time $\mathrm{k}$-t SENSE ( $\mathrm{p} 0.15$ ). There was however a small difference in LVEDV and thus, LVSV and LVEF, which did reach statistical significance $(\mathrm{p}<0.05)$. For cardiac gated versus standard real-time acquisitions, both RV and LV EDV and thus, SV and EF were significantly underestimated $(\mathrm{p}<0.05)$. For radial k-t versus standard real-time acquisitions only RVEF and LVEF were significantly different $(\mathrm{p}<0.05)$. The differences in the means were reflected in the biases from the Bland Altman analysis. For both $\mathrm{LV}$ and RV, bias in EDV, SV, and EF was less using radial k-t real-time compared to standard realtime. In addition, for all LV and RV volumes and ejection fraction, agreement with cardiac gated imaging was better with radial k-t real-time compared to standard real-time. (Figure 1.) 

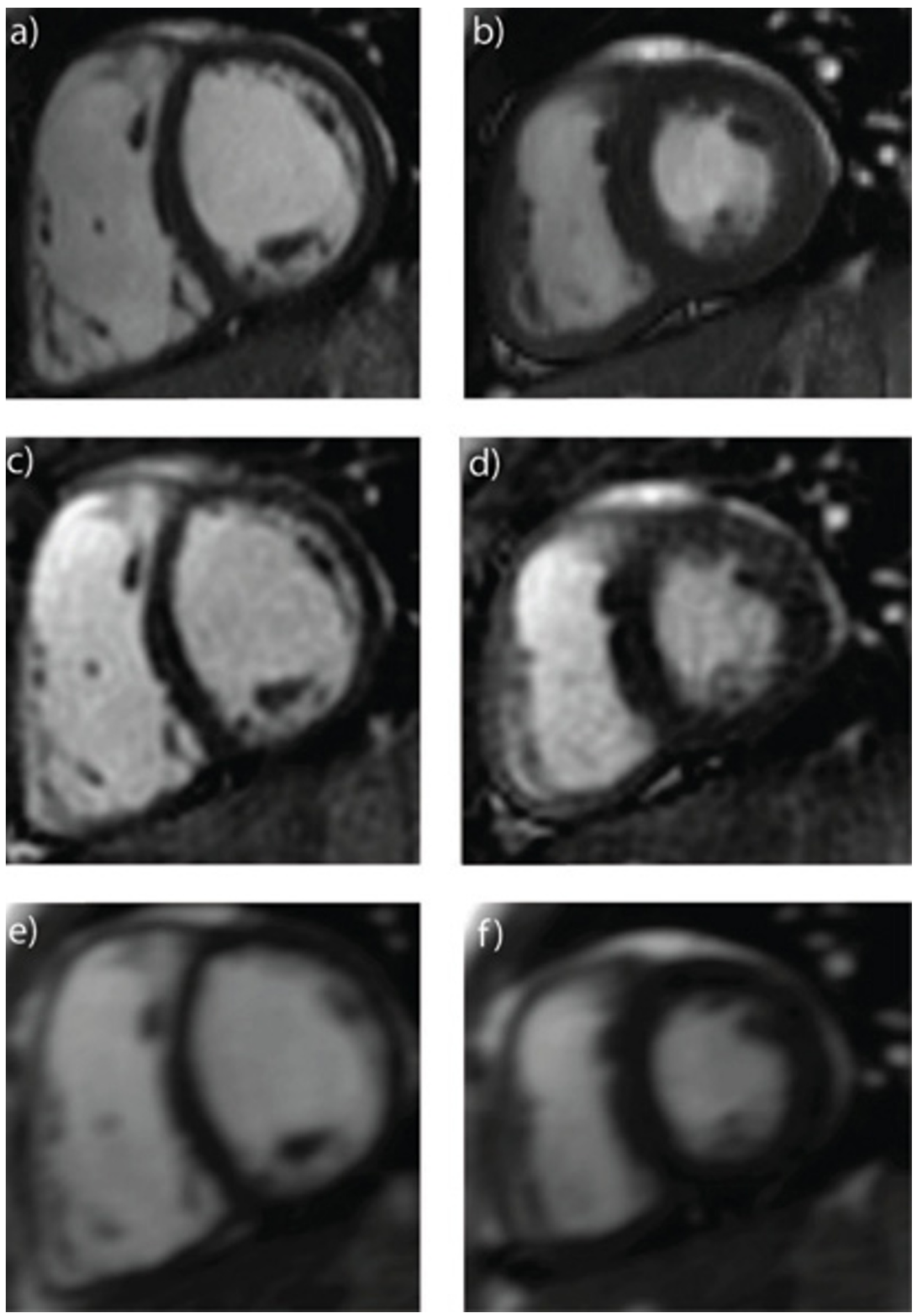

Figure I

Representative examples of cardiac gated images in diastole (a) and systole (b), radial k-t SENSE real-time images in diastole (c) and systole (d), and standard real-time images in diastole (e) and systole (f). 


\section{Conclusion}

Although real-time imaging has many benefits, its lower spatio-temporal resolution has hindered its use in the clinical environment. This has been particularly true in patients with congenital heart disease as the more difficult to segment $\mathrm{RV}$, is often the ventricle of interest. We have shown that radial k-t real-time allows accurate quantification of ventricular volumes and function in patients with congenital heart disease. In addition, image quality is superior to standard real-time, allowing better assessment of anatomy and motion. This opens up the possibility of performing a complete real-time exam in patients with congenital heart disease. This would be particularly useful in patients who are unable to hold their breath (i.e. paediatric patients), or have rhythm irregularities that compromise gated imaging.

Publish with Bio Med Central and every scientist can read your work free of charge

"BioMed Central will be the most significant development for disseminating the results of biomedical research in our lifetime. " Sir Paul Nurse, Cancer Research UK

Your research papers will be:

- available free of charge to the entire biomedical community

- peer reviewed and published immediately upon acceptance

- cited in PubMed and archived on PubMed Central

- yours - you keep the copyright

Submit your manuscript here:

http://www.biomedcentral.com/info/publishing_adv.asp 